\title{
ESTRATÉGIA EM ORGANIZAÇÕES: A PRODUÇÃO CIENTÍFICA BRASILEIRA ENTRE 2003 E 2011
}

STRATEGY IN ORGANIZATIONS: BRAZILIAN SCIENTIFIC PRODUCTION BETWEEN 2003 AND 2011

ESTRATEGIA EN ORGANIZACIONES: LA PRODUCCIÓN CIENTÍFICA BRASILEÑA ENTRE 2003 Y 2011

\author{
ROSILENE MARCON \\ Doutora \\ Universidade do Vale do Itajaí - Brasil \\ rmarcon@univali.br \\ RODRIGO BANDEIRA-DE-MELLO \\ Doutor \\ Fundação Getúlio Vargas - Brasil \\ rodrigo.bandeira.demello@fgv.br
}

Submetido em: 15/12/2015

Aprovado em: 13/04/2016

Doi: alcance.v23n2.p127-141

\begin{abstract}
RESUMO
Este artigo analisa nove anos (2003-2011) da pesquisa em estratégia no Brasil publicada nos principais periódicos de administração do país, e nos anais do EnANPAD, na divisão de Estratégia em Organizações (ESO), e do 3Es. A amostra de 1524 artigos foi submetida à análise de conteúdo utilizando um dicionário de termos para classificação de temas e metodologias de pesquisa. Os principais resultados indicam a continuidade da predominância das pesquisas exploratórias por meio de estudos de caso qualitativos e a tendência de crescimento da pesquisa nos temas sobre negócios internacionais e sobre sustentabilidade socioambiental e ética corporativa. Apesar desses temas de pesquisa alinharem-se ao contexto nacional e mundial, o excesso de estudos exploratórios não aprofunda nem adapta os paradigmas teóricos existentes à realidade nacional, muito menos contribui para ser inserido em um fórum de discussão internacional.
\end{abstract}

Palavras-chave: Estratégia em Organizações. Produção Científica. Metodologia de Pesquisa. Brasil.

\begin{abstract}
This study analyzes nine years (2003-2011) of research on strategy in Brazil, published in the country's main business administration periodicals in the country, and on the annals of the EnANPAD, in the division Strategy of Organizations (ESO) and the 3Es. The sample, consisting of 1524 articles, was submitted to content analysis using a dictionary of terms for classification of themes and research methodologies. The main results indicate the continued predominance of exploratory research, through qualitative case studies, and a tendency towards the growth of research on themes of international business, socienvironmental sustainability, and corporate ethics, Although these research themes are consistent with the national and global context, the abundance of exploratory research does not contribute or alter the existing theoretical paradigms of the Brazilian reality, nor does it contribute to the insertion of this community in the international forum of debate.
\end{abstract}

Keywords: Business Policy and Strategy. Research Themes. Methodology. Brazil.

Revista Alcance - Eletrônica - vol. 23 - n. 2 - abr./jun. 2016 


\section{RESUMEN}

Este artículo analiza nueve años (2003-2011) de investigación en estrategia en Brasil publicada en los principales periódicos de administración del país y en los anales del EnANPAD, en la división de Estrategia en Organizaciones (ESO) y del 3Es. La muestra de 1524 artículos fue sometida al análisis de contenido utilizando un diccionario de términos para la clasificación de temas y metodologías de investigación. Los principales resultados indican la continuidad del predominio de las investigaciones exploratorias por medio de estudios de caso cualitativos y la tendencia de crecimiento de la investigación en los temas sobre negocios internacionales y sobre sostenibilidad socioambiental y ética corporativa. A pesar de que estos temas de investigación coinciden con el contexto nacional y mundial, el exceso de estudios exploratorios no profundiza ni adapta los paradigmas teóricos existentes a la realidad nacional, y mucho menos contribuye para que sea incluido en un fórum de discusión internacional.

Palabras clave: Estrategia en Organizaciones. Producción Científica. Metodología de Investigación. Brasil.

\section{INTRODUÇÃO}

Apesar de estratégia ser um dos conceitos mais estudados e ensinados no mundo, ela ainda é pouco compreendida (RONDA-PUPO; GUERRA-MARTINS, 2012). Estudos como este, que analisa as publicações de uma comunidade em um determinado período, permite discutir, comparar e refletir sobre o desenvovimento e a consolidação das diversas dimensões temáticas que compõem o conceito de estratégia para essa comunidade. $\mathrm{Na}$ sequência de trabalhos precedessores que analisaram a produção científica em estratégia no Brasil (BERTERO; VASCONCELOS; BINDER, 2003; BIGNETTI; PAIVA, 2002; COLLA; MARTINS; KATO, 2012), este artigo analisa a produção científica de estratégia em organizações no Brasil no período de 2003 a 2011, a partir de todos os artigos de estratégia publicados nas principais revistas acadêmicas de administração no Brasil, sendo elas: a Revista de Administração de Empresas (RAE), a Revista de Administração Contemporânea (RAC), a Brazilian Administration Review (BAR), a Revista de Administração da Universidade de São Paulo (RAUSP) e a Brazilian Business Review (BBR). Foram ainda considerados na amostra os artigos apresentados na divisão acadêmica de estratégia em organizações (ESO) e publicados nos anais do Encontro Anual da Associação Nacional de Pós-graduação e Pesquisa em Administração (EnANPAD) e nos Anais do Enconto de Estudos em Estratégia (3E's), também da ANPAD. A escolha pelos congressos da ANPAD justifica-se pelo fato de eles serem os principais indicadores de tendências das publicações nacionais. Os dados da ANPAD evidenciam que a divisão ESO foi em 2012 a maior divisão acadêmica em número de artigos submetidos e apresentados.

Essa produção científica apresenta forte contraste com a produção estrangeira em estratégia no que diz respeito à sua delimitação temática. Da forma como se definiu aqui neste artigo, estratégia em organizações engloba temas que abrangem não somente o dito mainstream da área, baseado na competição, no mercado, nos recursos e no desempenho, mas também questões como o empreendedorismo, a relação com teoria das organizações, a ética e a sustentabilidade e a economia politica. Essa maior amplitude temática torna demasiadamente incompletos os modelos para análise da produção científica em estratégia os quais foram utilizados em artigos anteriores, como 0 modelo de Whitington (BERTERO; VASCONCELOS; BINDER, 2003). Por isso, neste artigo se decidiu construir um modelo de análise a partir dos temas definidos pela divisão ESO. Adotou-se uma abordagem empírica abdutiva: partiu-se dos artigos apresentados para criar e testar o modelo de análise. Esse modelo serviu como referência na análise de conteúdo para classificar os artigos da amostra total quanto à sua temática e metodologia. Isso permite que se tenha a utilização de um modelo conceitual amplo e legítimo para dar conta dessa produção.

\section{REFERENCIAL TEÓRICO}

\subsection{Análise da Produção Científica em Estratégia}

Bignetti e Paiva (2002) analisaram artigos publicados no EnANPAD classificados nas áreas de Organizações/Estratégia e Administração Estratégica no período de 1997 a 2001. Eles encontraram a predominância 
de uma visão determinística do ambiente, em que os tomadores de decisão aceitam o ambiente externo como dado e imutável e atuam internamente para compensar as influências externas, em oposição à visão de que a organização e o ambiente não são completamente separados e independentes, mas pertencentes a um mesmo continuum. Os autores destacaram também que Michael Porter foi um dos autores mais citados na amostra, além de uma baixa frequência de citação de autores nacionais.

Bertero, Vasconcelos e Binder (2003) apresentam levantamento da publicação em estratégia empresarial no Brasil entre os anos de 1991 a 2002, classificando os artigos com base: (a) na perspectiva teórica, utilizando o modelo de Richard Whittington (2001), (b) nas temáticas propostas pelos autores, (c) na metodologia empregada no artigo, (d) na instituição dos autores e (e) no número de autores por artigo. Bertero, Vasconcelos e Binder (2003) destacam que a nossa produção fez seleções e adequações à realidade estratégica das nossas empresas, apesar de não ficar alheia ao que ocorre internacionalmente. Contudo, demonstram que a produção nacional está concentrada em um número relativamente reduzido de autores e que esta produção não é prática, ou seja, não possui um caráter prescritivo para a área, com menção do que deve ser feito e também evitado. Finalmente, o estudo mostrou que a academia brasileira em estratégia é fortemente representada pelos autores da perspectiva clássica do modelo de Whittington, representada por nomes como Igor Ansoff $(1965 ; 1970 ; 1991)$ e Michael Porter (1980; 1981; 1985; 1996); seguida da perspectiva processual, de forte orientação Mintzberguiana (MINTZBERG, 1978). Colla, Martins e Kato (2012) também utilizaram o modelo Whittington ao descreverem de que forma ocorreu a evolução teórico-metodológica nos estudos brasileiros em Estratégia nos anos de 2000 a 2010.

Gosling e Gonçalves (2004) realizaram uma meta-análise dos trabalhos apresentados no EnANPAD de 1997 a 2002. Eles encontraram que nas análises dos métodos aplicados nos artigos há predomínio do uso de tratamento de dados de natureza qualitativa. Em suma, o predomínio das perspectivas clássicas e processual e 0 uso de metodologias qualitativas caracterizaram a pesquisa em estratégia no Brasil no final dos anos 1990, início dos anos 2000.

Os trabalhos internacionais se concentraram principalmente em analisar a estrutura e a evolução dos estudos em estratégia (BOWMAN; SINGH; THOMAS, 2002; HOSKINSSON; HITT; WAN; YIU, 1999; RAMOSRODRIGUEZ; RUIZ NAVARRO, 2004; FURRER; THOMAS; GOUSSEVSKAIA, 2008).

Hoskisson et al. (1999) associaram a evolução das teorias e das pesquisas em estratégia entre 1960 e final dos anos $90 \mathrm{com}$ o movimento de um pêndulo, considerando as características internas da firma em um extremo e as da indústria em outro. Furrer et. al. (2008) analisaram vinte e seis anos de pesquisa em estratégia e demonstraram 0 crescimento das seguintes palavras-chave: alianças, capacidades, reestruturação, internacional, empreendedorismo e inovação; e um decréscimo em fit, decisão, ambiente, planejamento, tipologias e missão. Guerras-Martín et al. (2014) complementaram a visão do pêndulo, destacando a existência de dois pêndulos em movimento constante, a tensão histórica entre os fatores internos e externos e a tensão entre um nível macro de análise - a empresa e o seu ambiente, e um nível mais micro - os indivíduos e suas relações dentro da firma.

\section{MÉTODO}

A partir de um levantamento bibliométrico, este estudo buscou analisar os principais artigos publicados no Brasil, enquadrados dentro da área de estratégia das organizações nos principais periódicos de administração do Brasil.

\subsection{Amostra}

Para analisar a produção científica brasileira em estratégia no período de 2003 a 2011, coletamos a produção correlata nos seguintes periódicos da área de administração no Brasil: Revista de Administração de Empresas (RAE), Revista de Administração Contemporânea (RAC), Brazilian Administration Review (BAR), Revista de Administração da Universidade de São Paulo (RAUSP) e Brazilian Business Review (BBR). Coletamos também os artigos apresentados na divisão de Estratégia em Organizações (ESO) e os publicados nos anais do EnANPAD e 3 Es no período. Com relação ao enquadramento dos artigos na área de estratégia, os artigos selecionados pelos 
congressos passam pelo crivo do coordenador da divisão e dos avaliadores. Os artigos não enquadrados como produção em estratégia tendem a não ser selecionados. Já para os artigos publicados nos periódicos, a seleção envolveu dupla conferência para decisão de considerá-lo ou não na amostra. A Tabela 1 apresenta a quantidade de artigos analisados em cada ano do período. 0 total da amostra é de 1524 artigos.

Tabela 1 - Base de artigos analisados na amostra

\begin{tabular}{l|l|l} 
Ano & Quantidade de artigos & $\%$ \\
\hline 2003 & 126 & 8,27 \\
\hline 2004 & 63 & 4,13 \\
\hline 2005 & 219 & 14,37 \\
\hline 2006 & 116 & 7,61 \\
\hline 2007 & 233 & 15,29 \\
\hline 2008 & 119 & 7,81 \\
\hline 2009 & 254 & 16,67 \\
\hline 2010 & 127 & 8,33 \\
\hline 2011 & 267 & 17,52 \\
\hline Total & 1524 & 100 \\
\hline
\end{tabular}

Fonte: Elaborada pelos autores.

Nota: os eventos 3Es ocorrem a cada dois anos (2003, 2005, 2007 e 2011), quando se nota o aumento significativo do número de artigos na amostra.

\subsection{Dimensões temáticas da pesquisa em estratégia}

Os artigos da amostra foram analisados quanto à sua dimensão temática e quanto à metodologia utilizada na pesquisa. $O$ problema central nesse tipo de análise diz respeito à delimitação do conceito de estratégia e às suas dimensões temáticas. Não há consenso sobre uma definição única do termo estratégia e não há uma linguagem comum entre os pesquisadores. Uma das prováveis razões se deve à diversidade na formação original dos pesquisadores, seja no marketing, na economia ou no comportamento organizacional, de forma que seus temas de interesse coincidem com seus respectivos campos de estudo originais (HAMBRICK, 2004; NAG; HAMBRICK; CHEN, 2007). Essa diversidade conceitual dificulta a compreensão e a caracterização da produção científica, visto que tornam os modelos de análise demasiadamente incompletos. Em um trabalho recente, publicado no Strategic Management Journal, Ronda-Pupo e Guerra-Martins (2012) analisaram os conceitos utilizados na academia entre 1962 e 2008 e encontraram mais de duzentas denominações para estratégia. Contudo, os autores concluem que os termos firma, ambiente, ações, recursos, objetivos e performance formam o núcleo da definição do conceito de estratégia, enquanto $\mathrm{Nag}$ et al. (2007) identificaram alguns componentes principais no conceito de estratégia: desempenho, firmas, ambiente, organização interna, gerentes/proprietários e recursos. De fato, de acordo com Hoskisson et al.(1999), a pesquisa em estratégia, que tinha seu foco inicial nas características das empresas, oscila, no final dos anos 70, para focar-se nos modelos ambientais porterianos. Finalmente, a partir dos anos 90, o foco volta-se para dentro da firma, com ênfase na visão baseada em recursos (RBV).

Se no exterior (notadamente na academia americana) o conceito de pesquisa em estratégia é aparentemente amplo, no Brasil essa amplitude é ainda maior. Essa nossa característica se reflete na atual estruturação temática da divisão acadêmica estratégia em organizações (ESO) da ANPAD. Em vez de focar-se em termos como firma, ambiente, recursos, objetivo e performance, os temas da divisão abrangem ética e responsabilidade socioambiental, questões institucionais e políticas da estratégia, empreendedorismo, e relação entre estratégia e teoria das organizações. O Quadro 1 descreve cada um dos 11 temas atuais de ESO. 
Quadro 1 - Dimensões Temáticas da Pesquisa em Estratégia em Organizações

\begin{tabular}{|c|c|}
\hline TEMAS & DESCRIÇÂOO \\
\hline $\begin{array}{l}\text { (1) Aspectos Teóricos e } \\
\text { Metodológicos da Vantagem } \\
\text { Competitiva }\end{array}$ & $\begin{array}{l}\text { Teoria da competição. Desafios teóricos e metodológicos da vantagem competitiva. } \\
\text { Modelagem, desenvolvimento, integração, especificação de conceitos e relações, e } \\
\text { operacionalização de construtos, da Resource-Based View, Knowledge-Based View, } \\
\text { Relational View, Value-Price-Cost, Paradigma porteriano (barreiras de mobilidade, } \\
\text { grupos estratégicos e sistema de atividades), Economia dos Custos de Transação e } \\
\text { perspectivas evolucionárias. Aspectos históricos das teorias de vantagem } \\
\text { competitiva. }\end{array}$ \\
\hline $\begin{array}{l}\text { (2) Formulação, } \\
\text { Implementação e Mudança } \\
\text { das Estratégias }\end{array}$ & $\begin{array}{l}\text { Ferramentas de gestão estratégica e análise de suas teorias subjacentes: } \\
\text { planejamento estratégico, cenários e técnicas de prospecção, Análises externa e } \\
\text { interna, análise estrutural das indústrias, identificação de recursos e competências, } \\
\text { análises dos stakeholders e fatores críticos de sucesso. Processos de tomada de } \\
\text { decisões estratégicas. Consultorias. Balanced Scorecard, mapas estratégicos e } \\
\text { ferramentas de visualização. Implementação e coordenação das estratégias. } \\
\text { Projetos de investimento estratégico e real options. A relação estratégia e estrutura } \\
\text { organizacional. Processos de mudança e adaptação estratégicas. O papel da } \\
\text { liderança e da equipe. Processos de formação de estratégias, estratégias } \\
\text { deliberadas e emergentes. }\end{array}$ \\
\hline $\begin{array}{l}\text { (3) Estratégia e } \\
\text { Conhecimento }\end{array}$ & $\begin{array}{l}\text { A natureza e a dinâmica das capacidades e das competências organizacionais. } 0 \\
\text { conceito de rotinas organizacionais. Conhecimento tácito e explícito. Aprendizagem } \\
\text { organizacional e vantagem competitiva. Competição, inovação e estratégias em } \\
\text { ambientes de rápidas mudanças tecnológicas. Dynamic capabilities. Aquisição, } \\
\text { desenvolvimento, transferência e replicação do conhecimento. Exploration, } \\
\text { explotation e path dependency. }\end{array}$ \\
\hline $\begin{array}{l}\text { (4) Estratégias Empresariais } \\
\text { e Corporativas }\end{array}$ & $\begin{array}{l}\text { Estratégias genéricas de posicionamento. A relação entre as estratégias } \\
\text { empresariais e funcionais (fit). O escopo horizontal e vertical da firma. Crescimento } \\
\text { da firma. Integração vertical. Estratégias de diversificação e gestão de portfólio. } \\
\text { Alocação de recursos. Fusões e aquisições. Business Groups. Governança } \\
\text { corporativa e conselho de administração. Estrutura de propriedade, controle e custos } \\
\text { de agência. }\end{array}$ \\
\hline $\begin{array}{l}\text { (5) Estratégia, } \\
\text { Sustentabilidade } \\
\text { Socioambiental e Ética } \\
\text { Corporativa }\end{array}$ & $\begin{array}{l}\text { Teorias, pesquisas e práticas sobre a concepção, implementação e avaliação de } \\
\text { estratégias para o alcance da sustentabilidade socioambiental em organizações com } \\
\text { ou sem fins lucrativos. Os fenômenos da responsabilidade social e da } \\
\text { responsabilidade ambiental. Estratégia e comportamentos éticos das organizações. } \\
\text { Comércio justo. }\end{array}$ \\
\hline $\begin{array}{l}\text { (6) Estratégia e } \\
\text { Empreendedorismo }\end{array}$ & $\begin{array}{l}\text { Abordagens teóricas e metodológicas do empreendedorismo. O(a) } \\
\text { empreendedor(a), seus modelos mentais e sua atuação estratégica. O } \\
\text { empreendedorismo e a inovação como fonte de vantagem competitiva. } \\
\text { Empreendedorismo em diferentes tipos e portes de organização. Estratégia, } \\
\text { crescimento da firma e o papel do(a) empreendedor(a). A atuação do(a) } \\
\text { empreendedor(a) no ambiente de negócios brasileiro. }\end{array}$ \\
\hline (7) Estratégia e Cooperação & $\begin{array}{l}\text { Estratégias coletivas e co-opetição. Redes e suas relações interorganizacionais. } \\
\text { Aglomerações territoriais, industriais e comerciais. Mecanismos e estruturas de } \\
\text { governança. Capital social. Confiança. Contratos relacionais. Inovação colaborativa, } \\
\text { co-criação, crowdsourcing e redes sociais digitais. Interação competitiva. } \\
\text { Reciprocidade. Teoria dos jogos. }\end{array}$ \\
\hline
\end{tabular}




\begin{tabular}{|c|c|}
\hline (8) Negócios Internacionais & $\begin{array}{l}\text { Processos e teorias de internacionalização. Modelos de gestão de empresas } \\
\text { internacionalizadas. Networks internacionais e internacionalização de clusters. } \\
\text { Empreendedorismo internacional. Padronização versus adaptação da oferta aos } \\
\text { mercados no exterior. Políticas públicas relacionadas à internacionalização. } \\
\text { Globalização e regionalização. Ambiente institucional do país hospedeiro. } \\
\text { Modelagem do desempenho internacional. Processo de internacionalização de } \\
\text { empresas brasileiras e de empresas de mercados emergentes. Tipologias de } \\
\text { empresas multinacionais brasileiras. Born Globals. }\end{array}$ \\
\hline $\begin{array}{l}\text { (9) Modelagem e } \\
\text { Mensuração do Desempenho }\end{array}$ & $\begin{array}{l}\text { Definição conceitual e abordagens teóricas do desempenho da firma. Apropriação } \\
\text { do valor. Teoria dos stakeholders vs. shareholders. Modelagem e operacionalização } \\
\text { do desempenho da firma, tanto como variável observável como latente. Questões } \\
\text { relacionadas ao uso de dados primários e secundários para mensuração do } \\
\text { desempenho. Drivers estratégicos do desempenho. Determinantes do desempenho } \\
\text { da firma em diferentes níveis de agregação (firma, indústria, grupo estratégico, } \\
\text { aglomeração, país, etc.). Dinâmica do desempenho e persistência de retornos infra e } \\
\text { supranormais. }\end{array}$ \\
\hline $\begin{array}{l}\text { (10) Perspectivas } \\
\text { Organizacionais e } \\
\text { Sociológicas da Estratégia }\end{array}$ & $\begin{array}{l}\text { Estratégia e teoria das organizações. Estratégia à luz do Institucionalismo } \\
\text { Sociológico, Dependência de Recursos, Escolha Estratégica, Ecologia das } \\
\text { Populações e perspectivas teóricas derivadas da Sociologia Econômica. } \\
\text { Abordagens da estratégia como prática social, discursiva ou cognitiva. Arquétipos, } \\
\text { configurações de organizações e ambiente, e o neo-contigencialismo. Ciclo de vida } \\
\text { das organizações e estratégia. Contexto social, histórico e político da estratégia nas } \\
\text { organizações. Cultura, simbolismo e estratégia. Construção do sentido, criação do } \\
\text { significado e ambiente construído na análise da estratégia. Racionalidade e gestão } \\
\text { estratégica. }\end{array}$ \\
\hline $\begin{array}{l}\text { (11) Estratégia, Governo e } \\
\text { Desenvolvimento }\end{array}$ & $\begin{array}{l}\text { Relações entre estratégia empresarial, sociedade e governo (em níveis nacional, } \\
\text { regional e global). Estratégia em organizações empresariais, governamentais, } \\
\text { sociais e híbridas. Teorias de globalização e de desenvolvimento em estratégia. } \\
\text { Teorização política e institucional em estratégia. Estratégia e regulação: } \\
\text { governamental, privada, e transnacional. Não mercado, teorias de mercado e } \\
\text { relações empresa-governo. Poder e política. Impactos de geopolítica e do mercado } \\
\text { de ideias em estratégia. Perspectivas críticas em estratégia e em temas emergentes } \\
\text { relacionados. }\end{array}$ \\
\hline
\end{tabular}

Fonte: Associação Nacional de Pos-Graduação e Pesquisa em Administração (ANPAD).

Argumentamos aqui, portanto, que os modelos utilizados nos artigos anteriores que analisaram a pesquisa em estratégia no Brasil não dão conta satisfatoriamente da nossa pluralidade temática. Por isso, adotamos como referência principal para nosso modelo de análise os 11 temas da divisão ESO na ANPAD no período considerado. Assumimos que a atual estruturação temática da divisão reflete a evolução do conceito de pesquisa em estratégia junto à comunidade nacional de pesquisadores.

Já com relação à metodologia, o problema da pluralidade foi relativamente simplificado. Seguindo orientações anteriores, utilizamos como referência quatro categorias: ensaio teórico, pesquisa quantitativa, pesquisa qualitativa, pesquisa quali-quanti, e três tipos de trabalhos empíricos: exploratórios, estudos de caso e explanatórios.

\subsubsection{Modelo de Análise}

Outro problema crucial para estudos de análise da produção científica diz respeito à arbitrariedade na designação de um artigo nesse ou naquele tema, e nessa ou naquela metodologia. Esse ponto é particularmente 
importante visto que a atual estruturação temática da divisão ESO está vigor somente desde 2011. Para atenuar esse problema, adotamos uma estratégia empírica abdutiva. Inicialmente, procedemos à análise de conteúdo dos termos mais utilizados nos artigos apresentados em cada tema do evento EnANPAD 2011. A análise identificou os termos mais frequentes nos artigos selecionados em cada tema. Em seguida, com base nessas frequências, elaboramos um dicionário de termos característicos de cada tema. Os dicionários foram ajustados e calibrados visando maximizar a eficiência na discriminação dos artigos em cada tema. Em média, o dicionário de um tema continha cerca de 15 termos. A calibragem ocorreu em diversos testes na mesma base de artigos (EnANPAD 2011). Com os dicionários calibrados, testamos sua capacidade de enquadrar os artigos em seus temas em outra base de dados, o evento 3Es 2011. Verificamos se nosso modelo previa com sucesso para qual tema determinado artigo fora submetido e fizemos os ajustes necessários.

O modelo final previa com considerável sucesso o tema principal e o secundário de cada artigo. A utilização de dois temas permite-nos aumentar as probabilidades de acerto na previsão do enquadramento, visto que naturalmente a maioria dos artigos perpassa mais de um tema. É comum acharmos artigos, por exemplo, que tratam de empreendedorismo e internacionalização, ou teorias das organizações e processo de formulação das estratégias. A nossa definição de tema principal foi aquele em que os termos de seu dicionário obtiveram conjuntamente a maior frequência. $\mathrm{E}$ o tema secundário, a segunda maior frequência na soma total da frequência de cada termo do dicionário. Como os termos de cada dicionário foram definidos de maneira única (equivalente à ortogonalidade dos temas), a utilização de dois temas (como sendo dois fatores) dá conta de grande parte da variação encontrada na classificação dos artigos. No processo de calibragem, contudo, devido à similaridade entre o tema (1) Aspectos Teóricos e Metodológicos da Vantagem Competitiva e o tema (9) Modelagem e Mensuração do Desempenho, bem como devido ao baixo número de artigos submetidos a esses dois temas, decidimos agrupá-los em um único tema.

Procedemos de maneira semelhante para classificar a metodologia de cada artigo. Construímos e testamos um dicionário de termos a partir da análise de uma amostra de artigos escolhidos aleatoriamente dentre os 1524 que compunham a amostra. Esse processo foi iterativo até que o modelo pudesse ser discriminante das sete categorias.

Todas as análises de conteúdo e categorização foram realizadas pelo Software Atlas.ti versão 7 . Ao todo, nos 1524 documentos analisados, foram geradas quase 500 mil referencias dos termos que compunham os dicionários dos temas e das categorias da metodologia.

\section{APRESENTAÇÃO E DISCUSSÃO DOS RESULTADOS}

A seguir apresentamos e discutimos os resultados sobre os temas e a metodologia dos artigos. A Tabela 2 apresenta a participação, ao longo dos anos, dos temas descritos no Quadro 1. 
Tabela 2 - Participação dos temas na amostra ao longo do período

\begin{tabular}{|l|l|l|l|l|l|l|l|l|l|l|}
\hline Ano & \multicolumn{1}{|l|}{ Temas } \\
\cline { 2 - 11 } & $1+9$ & 2 & 3 & 4 & 5 & 6 & 7 & 8 & 10 & 11 \\
\hline 2003 & 6,35 & 16,67 & 16,67 & 12,70 & 11,90 & 2,38 & 4,76 & 10,32 & 7,94 & 10,32 \\
\hline 2004 & 15,87 & 14,29 & 14,29 & 12,70 & 6,35 & 0,00 & 11,11 & 11,11 & 6,35 & 7,94 \\
\hline 2005 & 11,42 & 8,22 & 13,24 & 12,33 & 6,85 & 8,22 & 8,22 & 19,18 & 5,94 & 6,39 \\
\hline 2006 & 8,62 & 7,76 & 4,31 & 10,34 & 3,45 & 16,38 & 6,03 & 25,86 & 10,34 & 6,90 \\
\hline 2007 & 9,44 & 8,58 & 7,30 & 7,30 & 8,15 & 8,58 & 8,58 & 28,76 & 9,01 & 4,29 \\
\hline 2008 & 8,40 & 10,92 & 4,20 & 5,04 & 3,36 & 13,45 & 13,45 & 31,09 & 6,72 & 3,36 \\
\hline 2009 & 7,48 & 7,09 & 7,48 & 5,91 & 18,11 & 5,91 & 9,45 & 21,65 & 11,02 & 5,91 \\
\hline 2010 & 14,96 & 9,45 & 6,30 & 7,09 & 18,90 & 3,15 & 10,24 & 18,11 & 7,87 & 3,94 \\
\hline 2011 & 9,74 & 10,86 & 6,37 & 4,12 & 14,61 & 4,87 & 7,49 & 22,47 & 10,11 & 9,36 \\
\hline
\end{tabular}

Fonte: Elaborada pelos autores.

Nota: os valores nas células referem-se ao percentual de artigos de um determinado tema em um determinado ano, em relação ao total de artigos produzidos naquele ano.

Nota-se que o tema negócios internacionais e sustentabilidade socioambiental e ética corporativa apresenta um forte crescimento ao longo do tempo. Esses resultados acompanham parcialmente os encontrados no mesmo período por Bach (2011), ao constatar que os autores na área de estratégia no Brasil buscam temas como internacionalização e desempenho organizacional. A Figura 1 apresenta graficamente os dados da Tabela 2.

Figura 1 - Comportamento dos temas ao longo do tempo

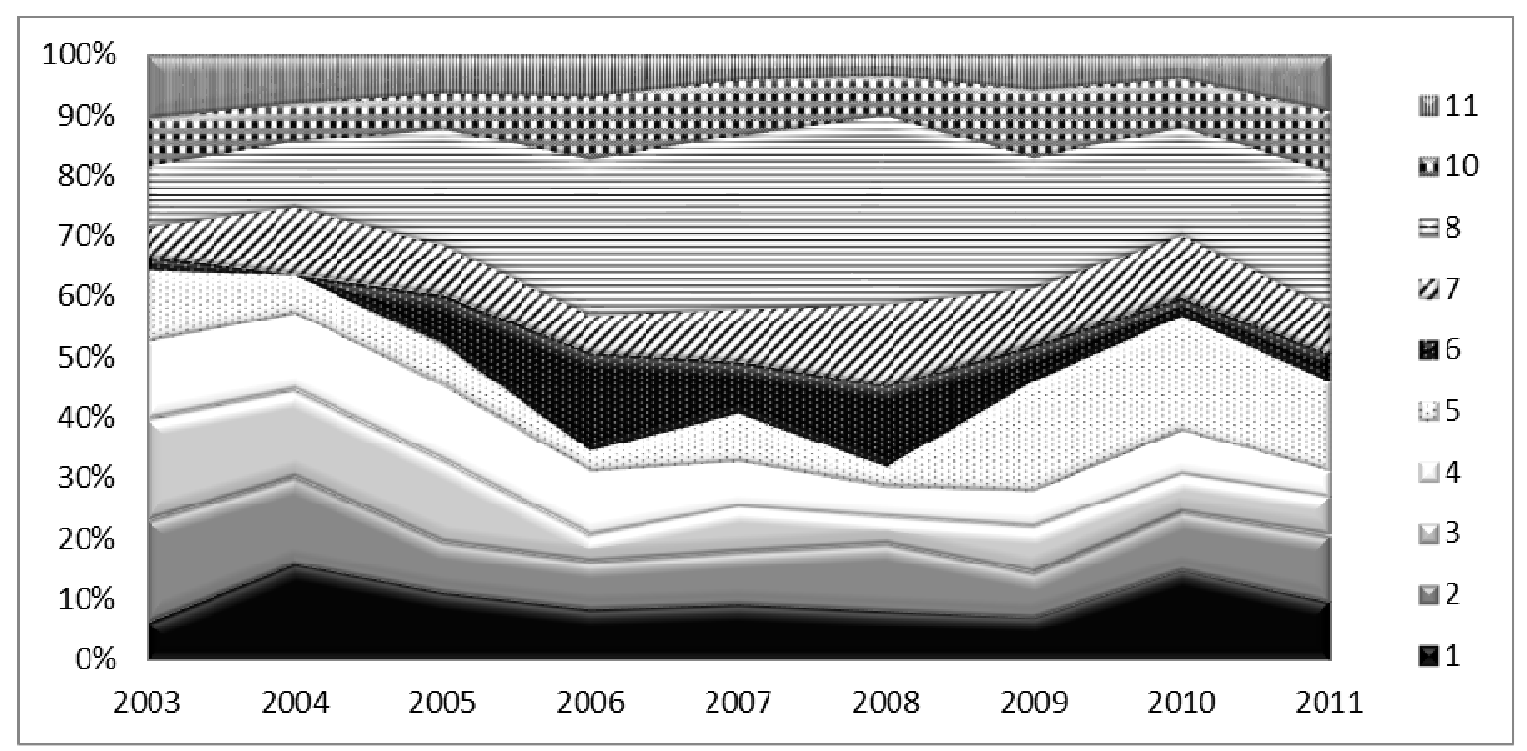

Fonte: Elaborada pelos autores. 
Quando comparamos nossos resultados com aqueles realizados em décadas anteriores, verificamos que alguns temas tiveram uma redução significativa, como gestão do conhecimento (BIGNETTI; PAIVA, 2002) e estratégias empresariais e corporativas (GOSLING; GONÇALVES, 2004). Uma provável justificativa para essa evidência pode ser inferida a partir da Tabela 3, que mostra a co-ocorrência dos temas. Podemos observar que 0 tema (3) estratégia e conhecimento é tema secundário importante para quase todos os demais temas, com destaque para tema (6) empreendedorismo, (7) redes e cooperação e (8) negócios internacionais. Isso mostra que a dimensão do conhecimento na pesquisa em estratégia continua relevante e interagindo com outras dimensões importantes.

O mesmo já não pode ser dito do tema (4) estratégias empresariais e corporativas. Esse tema somente é importante tema secundário para o tema $(1+9)$ que aborda a modelagem da vantagem competitiva e do desempenho. Uma análise mais focada da publicação do tema (4) mostra grande influência do paradigma porteriano. Esses resultados em conjunto nos levam a crer que a predominância do modelo porteriano, observada nos estudos em períodos anteriores, está perdendo força. Resultado semelhante é apresentado por Sehnem, Lazzarotti e Bandeira-de-Mello (2010). Esses autores mostram que o paradigma porteriano vem perdendo força ao longo da década. 0 desdobramento da Tabela 3, ano a ano, mostra que em 2003 e 2004 os temas com maior intersecção foram 0 de estratégias empresariais e corporativas com 0 tema sobre vantagem competitiva e mensuração do desempenho, próximos, de fato, da perspectiva clássica da estratégia indicada nos estudos de períodos anteriores. No entanto, a partir de 2005, a intersecção do tema negócios internacionais com estratégia, governo e desenvolvimento começa a ter destaque, mantendo-se até 2011.

É interessante observar ainda que, com relação aos dois temas que mais cresceram, negócios internacionais, e sustentabilidade socioambiental e ética corporativa, ambos possuem como tema secundário principal o tema (11) que aborda as questões institucionais de governo, globalização e desenvolvimento, as estratégias de organizações híbridas, como as organizações não governamentais (ONG), e uma visão crítica sobre a estratégia. Isso sugere um aumento da amplitude temática da pesquisa em estratégia à medida que tende a considerar questões que transcendem a empresa focal em um mercado, mas considera elementos institucionais do ambiente externo, diferentes formas organizacionais e atores ambientais.

Outra evidência relevante é quanto ao tema (10) perspectivas organizacionais e sociológicas da estratégia. Analisando a Tabela 2, nota-se que ele permaneceu estável ao longo do período, ou seja, imune a possíveis modismos. Isso mostra que a questão organizacional e sociológica é uma dimensão importante e fortemente enraizada na tradição de pesquisa em estratégia no Brasil. Além disso, quase a metade de sua produção é feita em conjunto com o tema (5) sustentabilidade socioambiental e ética corporativa, que apresenta tendência de forte crescimento.

Finalmente, o tema (6) sobre empreendedorismo apresenta forte crescimento no meio do período seguido de um decréscimo no final do período analisado. Uma provável explicação pode ser a divisão da produção sobre empreendedorismo em outros fóruns de pesquisa não considerados na amostra, como o Encontro sobre Empreendedorismo e Gestão de Pequenas Empresas (EGEPE). 
Tabela 3 - Temas principal e secundário dos artigos analisados

\begin{tabular}{|c|c|c|c|c|c|c|c|c|c|c|}
\hline TEMAS & Secur & dário & & & & & & & & \\
\hline Principal & $1+9$ & 2 & 3 & 4 & 5 & 6 & 7 & 8 & 10 & 11 \\
\hline $1+9$ & & 6,71 & 12,75 & 32,89 & 8,72 & 0,00 & 4,70 & 22,15 & 7,38 & 4,70 \\
\hline 2 & 6,71 & & 17,45 & 7,38 & 18,79 & 4,70 & 3,36 & 6,04 & 16,78 & 18,79 \\
\hline 3 & 13,08 & 13,08 & & 13,85 & 6,15 & 2,31 & 5,38 & 6,15 & 9,23 & 30,77 \\
\hline 4 & 41,32 & 6,61 & 11,57 & & 4,13 & 1,65 & 5,79 & 10,74 & 6,61 & 11,57 \\
\hline 5 & 6,47 & 17,65 & 1,76 & 7,06 & & 2,35 & 3,53 & 12,35 & 19,41 & 29,41 \\
\hline 6 & 10,19 & 7,41 & 24,07 & 5,56 & 19,44 & & 9,26 & 6,48 & 2,78 & 14,81 \\
\hline 7 & 16,03 & 5,34 & 20,61 & 10,69 & 6,11 & 2,29 & & 9,16 & 9,16 & 20,61 \\
\hline 8 & 13,47 & 2,69 & 17,96 & 12,28 & 5,09 & 2,99 & 9,88 & & 8,68 & 26,95 \\
\hline 10 & 5,26 & 15,04 & 7,52 & 3,76 & 42,11 & 0,75 & 8,27 & 3,01 & & 14,29 \\
\hline 11 & 6,06 & 9,09 & 14,14 & 8,08 & 20,20 & 2,02 & 13,13 & 15,15 & 12,12 & \\
\hline
\end{tabular}

Fonte: Elaborada pelos autores baseado nos dados da pesquisa.

Nota: os valores nas células referem-se ao percentual de artigos do tema principal em conjunto com o tema secundário em relação ao total de artigos do tema principal naquele ano.

Ao analisar as metodologias utilizadas, os resultados mostram que não houve alterações significativas quando comparadas aos resultados das pesquisas de períodos anteriores. A Tabela 4 mostra os resultados encontrados para as quatro categorias da metodologia.

Tabela 4 - Metodologia utilizada nos artigos da amostra ao longo do período

\begin{tabular}{|l|l|l|l|l|}
\hline Ano & Qualitativa & Quantitativa & Quali-Quanti (QQ) & Ensaio Teórico \\
\hline 2003 & $46,03 \%$ & $23,02 \%$ & $7,94 \%$ & $23,02 \%$ \\
\hline 2004 & $52,38 \%$ & $33,33 \%$ & $1,59 \%$ & $12,70 \%$ \\
\hline 2005 & $48,86 \%$ & $22,83 \%$ & $10,05 \%$ & $18,26 \%$ \\
\hline 2006 & $46,55 \%$ & $23,28 \%$ & $11,21 \%$ & $18,97 \%$ \\
\hline 2007 & $49,79 \%$ & $27,47 \%$ & $7,30 \%$ & $15,45 \%$ \\
\hline 2008 & $42,02 \%$ & $33,61 \%$ & $10,92 \%$ & $13,45 \%$ \\
\hline 2009 & $47,64 \%$ & $29,53 \%$ & $7,09 \%$ & $15,75 \%$ \\
\hline 2010 & $40,94 \%$ & $25,20 \%$ & $11,81 \%$ & $22,05 \%$ \\
\hline 2011 & $48,31 \%$ & $28,09 \%$ & $8,61 \%$ & $14,98 \%$ \\
\hline
\end{tabular}

Fonte: Elaborada pelos autores. 
Nota: os percentuais nas células referem-se ao número de artigos que utilizou uma determinada metodologia no ano em relação ao total de artigos publicados naquele ano.

A Figura 2 apresenta a análise gráfica da Tabela 4. Percebe-se a larga predominância da metodologia qualitativa e uma estabilidade da proporção entre elas ao longo do tempo. Quase a metade do total de artigos publicados (considerando os ensaios teóricos) utiliza a abordagem qualitativa. Esse é um número expressivo e que destoa da característica comum da publicação nas revistas estrangeiras de primeira linha, na qual uma pequena minoria é qualitativa. Os resultados corroboram os achados de Colla et al. (2012), que também evidenciaram uma predominância de estudos qualitativos na produção cientifica de estratégia entre os anos de 2000 a 2010 e os de Bertero e outros (2003) no período de 1991-2002. Podemos concluir facilmente que a metodologia qualitativa apresenta grande resiliência na pesquisa em estratégia, apesar das pressões por publicação internacional.

Figura 2 - Comportamento da metodologia ao longo do tempo

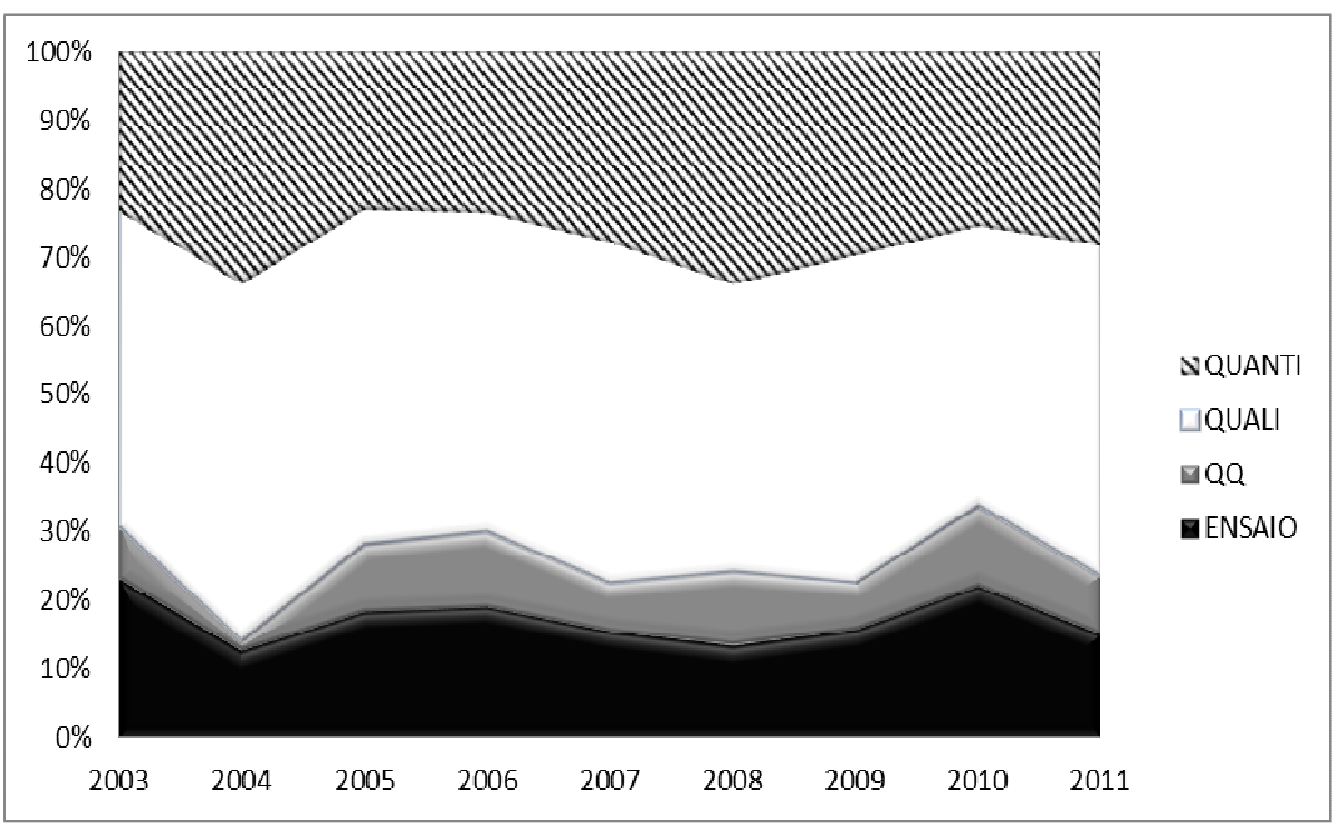

Fonte: Elaborada pelos autores.

A Tabela 5 especifica os resultados da análise da metodologia por tema, a partir das duas classificações realizadas nesta pesquisa. Em um primeiro momento classificamos os artigos em ensaios teóricos, quantitativos, qualitativos, e quali-quanti (QQ). Em seguida, com o objetivo de verificar o amadurecimento metodológico da área, agrupamos os trabalhos empíricos em exploratório, estudos de caso e explanatórios. 
Tabela 5 - Metodologias utilizadas e tipos de artigos empíricos por tema

\begin{tabular}{l|l|l|l|l|l|l|l|l}
\hline \multirow{2}{*}{ TEMA } & \multirow{2}{*}{$\%$} & \multicolumn{2}{l}{ TIPOS } & \multicolumn{2}{l}{ EMPIRICOS } \\
\cline { 3 - 9 } & & $\begin{array}{l}\text { Ensaio } \\
\text { Teórico }\end{array}$ & $\begin{array}{l}\text { Quali- } \\
\text { Quanti }\end{array}$ & Quali & Quanti & Exploratório & $\begin{array}{l}\text { Estudo de } \\
\text { Caso }\end{array}$ & Explanatório \\
\hline $1+9$ & 9,78 & 17,45 & 11,41 & 14,77 & 56,38 & 35,57 & 20,81 & 25,50 \\
\hline 2 & 9,78 & 15,44 & 8,72 & 57,05 & 18,79 & 42,95 & 57,72 & 6,71 \\
\hline 3 & 8,53 & 16,92 & 10,77 & 46,92 & 25,38 & 43,85 & 46,92 & 5,38 \\
\hline 4 & 7,94 & 21,49 & 5,79 & 52,89 & 19,83 & 36,36 & 38,84 & 15,70 \\
\hline 5 & 11,15 & 19,41 & 7,06 & 51,18 & 22,35 & 27,65 & 44,71 & 8,24 \\
\hline 6 & 7,09 & 13,89 & 8,33 & 56,48 & 21,30 & 54,63 & 37,04 & 8,33 \\
\hline 7 & 8,6 & 14,50 & 8,40 & 56,49 & 20,61 & 33,59 & 54,96 & 7,63 \\
\hline 8 & 21,92 & 14,37 & 8,38 & 39,82 & 37,43 & 30,84 & 48,20 & 23,95 \\
\hline 10 & 8,73 & 21,80 & 10,53 & 54,89 & 12,78 & 29,32 & 42,86 & 7,52 \\
\hline 11 & 6,5 & 18,18 & 7,07 & 60,61 & 14,14 & 29,29 & 46,46 & 10,10 \\
\hline
\end{tabular}

Fonte: Elaborada pelos autores.

Nota: nos percentuais dos estudos empíricos, ressalta-se que as três classificações não são mutuamente excludentes, já que é possivel um artigo utilizar o design de caso e ser também exploratório ou explanatório.

Na maioria dos temas a predominância é a pesquisa qualitativa, com estudo de caso exploratório. As exceções são os temas $(1+9)$ sobre modelagem da vantagem competitiva e do desempenho, o que faz todo sentido dado à natureza das questões colocadas nesse tema. No entanto, dentre os empíricos, ainda há a preponderância de estudos exploratórios ou descritivos em relação aos estudos explanatórios ou causais. Outro destaque é para o tema (8) sobre negócios internacionais, o qual apresenta uma relação paritária entre metodologias qualitativas e quantitativas.

A Tabela 6 mostra os resultados dos trabalhos empíricos, permitindo uma análise longitudinal das metodologias dos trabalhos empíricos. 
Tabela 6 - Descrição dos estudos empíricos por ano

\begin{tabular}{|l|c|c|c|}
\hline Anos & EXPLORATORIA & ESTUDO DE CASO & EXPLANATÓRIA \\
\hline 2003 & 35,05 & 42,27 & 12,37 \\
\hline 2004 & 36,36 & 49,09 & 25,45 \\
\hline 2005 & 43,58 & 51,40 & 10,61 \\
\hline 2006 & 43,62 & 52,13 & 14,89 \\
\hline 2007 & 46,19 & 53,81 & 14,72 \\
\hline 2008 & 35,92 & 52,43 & 23,30 \\
\hline 2009 & 44,39 & 54,67 & 17,76 \\
\hline 2010 & 37,37 & 52,53 & 17,17 \\
\hline 2011 & 44,05 & 58,15 & 16,74 \\
\hline
\end{tabular}

Fonte: Elaborada pelos autores.

Ano a ano os resultados mostram a estabilidade dos resultados. Novamente não se nota uma alteração no padrão de predominância de estudos de caso qualitativos e exploratórios em relação aos estudos de design explanatório-causal. Enquanto a pesquisa exploratória pode representar uma abertura maior para novas possibilidades de pesquisa, sua preponderância em relação a designs causais permite-nos inferir que a produção científica em estratégia no Brasil aborda as teorias da estratégia de maneira apenas superficial. Designs causais permitem especificar, estender e adaptar paradigmas teóricos para novas evidências, inclusive as particularidades do Brasil. Por isso, o excesso de trabalhos exploratórios impede esse movimento de aprofundamento dos paradigmas. Gosling e Gonçalves (2004) encontraram no seu estudo, que envolveu o período de 1997 a 2002, uma ausência absoluta de pesquisas causais, de acordo com seu critério.

Ao contrário das publicações internacionais, a produção acadêmica em estratégia no Brasil, em nenhum período do desenvolvimento da área, teve a predominância de pesquisas explanatórias. Esses resultados são intrigantes dado que é comum encontrar nas pesquisas nacionais uma citação elevada de autores internacionais, porém na aplicação dos métodos não seguimos as tendências vindas de fora do país. Isso torna nossa produção mais distante da característica da produção científica publicada nos periódicos de primeira linha estrangeiros. Cabenos, portanto, indagar quais são os processos geradores dessas evidências.

\section{CONCLUSÕES}

Este artigo faz uma análise da produção científica de estratégia no Brasil no período de 2003 a 2011, englobando todos os artigos de estratégia publicados nas principais revistas acadêmicas de administração no Brasil e nos dois principais eventos com artigos da área: EnANPAD e 3Es. Ao todo foram analisados 1524 trabalhos ao longo de 11 dimensões temáticas e sete categorias metodológicas. Concluímos que a pesquisa em estratégia no Brasil continua essencialmente exploratória e baseada em estudos de caso qualitativos. Essa característica perpassa todos os temas pesquisados, com exceção dos temas sobre a modelagem da vantagem competitiva e do desempenho e sobre negócios internacionais.

Se por um lado os estudos exploratórios são mais adequados a compreender a realidade da estratégia de nossas empresas, com constataram Bertero, Vasconcelos e Binder (2003) em seu estudo na década anterior, sua larga preponderância mostra uma fase ainda embrionária e pré-paradigmática da nossa pesquisa em estratégia. 0 paradoxo dessas evidências é que tradicionalmente os autores estrangeiros (e seus paradigmas) são mais citados do que os nacionais, porém os métodos destinados a desenvolver esses paradigmas não são utilizados. Fica, 
portanto, uma inconsistência entre a realidade empírica e uma revisão bibliográfica desconectada, no caso de estudos exploratórios, ou sem aderência à realidade local, no caso dos estudos descritivos.

Os resultados ainda mostram um crescimento das pesquisas que envolvem negócios internacionais e sustentabilidade socioambiental e ética corporativa. De fato, esses temas refletem os fenômenos marcantes do país na década, como as multinacionais brasileiras e o apelo socioambiental. A busca de nossas empresas por novos mercados exigiu também dos estudos de estratégias empresariais esta mudança de foco de investigação. A crescente importância das economias emergentes se reflete em um aumento nas pesquisas em estratégia sobre 0 tema, não só no Brasil, mas também nas publicações internacionais (WRIGHT et al., 2005). 0 tema para 2012 da conferência anual da SMS (Strategic Management Society) foi Strategy in Transition em função das mudanças de sistemas políticos, econômicos e institucionais, reflexo do aumento dos mercados emergentes em todo o mundo. Apresenta-se, portanto, uma oportunidade para inserirmo-nos em um discurso mais amplo. Mas esse percurso passa por evoluirmos em termos metodológicos e alcançarmos um equilíbrio entre exploração e aprofundamento dos paradigmas.

Uma das limitações deste artigo é o intervalo de tempo da análise, pois foi considerado este período em função dos temas e suas descrições dentro da área de estratégia em administração da Associação Nacional de PósGraduação e Pesquisa em Administração (ANPAD)para melhor padronização.

\section{REFERÊNCIAS}

ANSOFF, H. I. Corporate strategy. Harmondsworth: Penguin, 1965.

ANSOFF, H. I. Critique of Henry Mintzberg's "The design school". Strategic Management Journal, n. 12, p. 449-61, 1991.

ANSOFF, H. I. Toward a strategic theory of the firm. In: Ansoff, H. I. (Org). Business strategy. London: Penguin, p. 11-40. 1970.

BACH, T. M. Inserção de Pesquisadores Entrantes na Área de Estratégia: Análise das Relações de Autoria e Temas Estudados no Período de 1997-2010. In: ENCONTRO NACIONAL DA ASSOCIAÇÃO NACIONAL DOS PROGRAMAS DE PÓS-GRADUAÇÃO EM ADMINISTRAÇÃO, 35, 2011, Rio de Janeiro. Anais. Rio de Janeiro: ANPAD, 2011.

BERTERO, C. O.; VASCONCELOS, F. C de; BINDER, M. P. Estratégia Empresarial: A Produção Científica Brasileira entre 1991 e 2002. Revista de Administração de Empresas (RAE), v. 43, n. 4, p. 48-62, 2003.

BIGNETTI, L. P.; PAIVA, E. L. Ora (Direis) Ouvir Estrelas!: Estudo das Citações de Autores de Estratégia na Produção Acadêmica Brasileira. Revista de Administração Contemporânea (RAC), v. 6, n.1, p. 105-125, 2002.

BOWMAN, E.H.; SINGH, H.; THOMAS, H. The domain of strategic management: history and evolution. In: PETTIGREW, A., THOMAS, H.; WHITTINGTON, R. (Eds.) Handbook of Strategy and Management. London: Sage. 2002.

COLLA, E. J.; MARTINS, T. S.; KATO, H. T. A Produção Científica Brasileira em Estratégia entre os anos $2000 \mathrm{e}$ 2010. In: ENCONTRO NACIONAL DA ASSOCIAÇÃO NACIONAL DOS PROGRAMAS DE PÓSGRADUAÇÃO EM ADMINISTRAÇÃO, 36, Rio de Janeiro. Anais. Rio de Janeiro: ANPAD, 2012.

FURRER, O.; THOMAS, H.; GOUSSEVSKAIA; A. The Structure and Evolution of the Strategic Management Field: A Content analysis of 26 Years of Strategic Management Research International Journal of Management Reviews, v. 10, Is. 1, p. 1. 2008.

GOSLING, M.; GONÇALVES, C. A. Ideias Metodológicas dos Autores de Estratégia dos Enanpads: uma MetaAnálise. Revista Eletrônica de Administração (REAd), v. 10, n. 5, p. 1-26, 2004.

GUERRAS-MARTÍNA, L. A.; MADHOK. A.; MONTORO-SÁNCHEZ, A. The evolution of strategic management research: Recent trends and current directions. BRQ Business Research Quarterly, v. 17, n. 6, 2014 
HAMBRICK, D. The disintegration of strategic management: it's time to consolidate our gains. Strategic Organization, v. 2, n. 1, p. 91-98, 2004.

HOSKINSSON, R. E.; HITT, M. A.; WAN, W. P.; YIU, D. Theory and Research in Strategic management: swings of a pendulum. Journal of Management, v. 25, n. 3, p. 417- 456, 1999.

MINTZBERG, H. Patterns in strategy formation (in strategy formulation). Management Science, v. 24, n. 9, p. 93448, 1978.

NAG, R.; HAMBRICK, D.C.; CHEN M. J. What is strategic management, really? Inductive derivation of a consensus definition on the field. Strategic Management Journal, v. 28, n.9, p. 935-955, 2007.

PORTER, M. Competitive advantage - creating and sustaing superior performance. New York: Free Press, 1985.

PORTER, M. Competitive strategy - techniques for analyzing industries and competitors. New York : Free Press, 1980.

PORTER, M. The contributions of industrial organization to strategic management. Academy of Management Review, v. 6, n. 4, p. 609-20, 1981.

PORTER, M. What is strategy. Harvard Business Review, p. 61-78, Nov./Dec. 1996.

RAMOS-RODRIGUEZ, A.R., RUIZ-NAVARRO, J. Changes in the intellectual structure of strategic management research: a bibliometric study of the Strategic Management Journal, 1980-2000. Strategic Management Journal, n.25, p. 981-1004. 2004.

RONDA-PUPO, G. A; GUERRAS-MARTIN, L. A. Dynamics of the evolution of the strategy concept 1962-2008: a coword analysis. Strategic Management Journal, v. 33, n. 2, p. 162-188, February 2012.

SEHNEM, S.; LAZZAROTTI, F.; BANDEIRA-DE-MELLO, R. Uma análise longitudinal da utilização do paradigma Porteriano no Brasil. Brazilian Business Review. v. 7, n. 2, 2010.

WHITTINGTON, R. What is strategy - and does it matter? London: Tomson Learning, 2001.

WRIGHT, M.; FILATOTCHEV, I.; HOSKISSON, R. E.; PENG, M. W. Strategy Research in Emerging Economies: challenging the conventional wisdom. Journal of Management Studies, n.42, p. 1-33, 2005. 